\title{
ULTRASONIC STUDY OF THERMAL HYSTERESIS IN HELICAL ANTIFERROMAGNETIC Dy
}

\author{
lu. Liubimova ${ }^{1}$, K. Sapozhnikov ${ }^{1,2}$, V. Nikolaev ${ }^{1,2}$, M.-LI. Corró ${ }^{3}$ and S. Kustov ${ }^{3}$ \\ ${ }^{1}$ ITMO University, Kronverksky pr. 49, St. Petersburg, 197101, Russia \\ ${ }^{2}$ loffe Institute, Russian Academy of Sciences, ul. Politekhnicheskaya 26, St. Petersburg, 194021 Russia \\ ${ }^{3}$ Department of Physics, University of Balearic Islands, Cra. Valldemossa km 7.5, \\ Palma de Mallorca E07122, Spain
}

Received: November 11, 2018

\begin{abstract}
High-resolution ultrasonic mechanical spectroscopy technique has been used to study the nature and dynamics of lattice defects and magnetic domain walls in the helical-type antiferromagnetic phase during thermal cycling of polycrystalline Dy samples between 80 and $210 \mathrm{~K}$. Effects of the lowest temperature of thermal cycles, applied magnetic field and cooling/ heating rate on the ultrasonic absorption and Young's modulus have been investigated. A strong influence of cooling/heating rate on the ultrasonic absorption is found over the temperature range between the Néel temperature, ca. $178 \mathrm{~K}$, and approximately $145 \mathrm{~K}$, confirming the existence of a new category of magnetomechanical damping - transitory ultrasonic absorption related to translational motion of domain walls. A strong increase of the ultrasonic absorption below approximately $140 \mathrm{~K}$ is attributed to the formation of nuclei of ferromagnetic phase, presumably stabilized by such lattice defects as dislocations. The effect of applied magnetic field on ultrasonic absorption also emerges below $140 \mathrm{~K}$ and is ascribed to the appearance of the net magnetization due to ferromagnetic nuclei. We argue that these nuclei are responsible for the controversial thermal hysteresis of elastic and anelastic properties, which is strongly promoted by decreasing the temperature of thermal cycles.
\end{abstract}

\section{INTRODUCTION}

Antiferromagnetic (AFM) materials have recently attracted much attention due to promising applications in spintronics [1,2]. AFM multilayered structures demonstrate tunneling anisotropic magnetoresistance $[2,3]$ and are promising for applications in electric current-controlled magnetic memory, resistant to external magnetic fields and protected from unwanted readout using magnetic probes $[3,4]$. Antiferromagnets are characterized by much faster spin dynamics than in ferromagnets (FM). Theoretical predictions show also that domain wall (DW) motion in AFM can reach velocities orders of magnitude higher than in ferromagnets $[4,5]$. However, studying DW dynamics in AFM materials is much more complicated than in FM, since the motion of DWs in AFM does not produce net magnetization change. We have shown recently that high resolution mechanical spectroscopy technique is efficient in studying defect and DW dynamics in polycrystalline AFM Dy. In particular, a coexistence of fast and slow DW dynamics, scaling with $\dot{T}$-dot and showing logarithmic isothermal kinetics, respectively, was reported [6]. Moreover, using acoustic technique, it has been shown that low-frequency DW fluctuation observed in Dy by photon correlation spectroscopy [7] are due to their Brownian-like motion provoked by spin fluctuations over the temperature range of critical slowing down [6]. Dysprosium is a classical model material with attractive

Corresponding author:Yu. Liubimova, e-mail: liubimovaiuliia@corp.ifmo.ru 
magnetic properties [8,9], possessing a complicated temperature-magnetic field phase diagram [10-14]. Dy is paramagnetic above the Néel temperature $T_{N}$ $=178.5 \mathrm{~K}$. In absence of magnetic field, Dy orders antiferromagnetically between the $T_{\mathrm{N}}$ and $T_{\mathrm{C}} \sim 85 \mathrm{~K}$ : magnetic moments are confined to the basal planes of hexagonal lattice and form a helicoidal structure with a pitch varying [15] from ca. $43.2^{\circ}$ at $T_{N}$ to $26.5^{\circ}$ close to $T_{C}$. One of the long-standing problems is thermal hysteresis of magnetic, elastic and anelastic properties of Dy [16-20], provoked by thermal cycling. Recently, studying reversible inverse magnetostriction and AC magnetic susceptibility of polycrystalline Dy [21], we have argued that thermal hysteresis disappears abruptly at a temperature of the Villari point at ca. 166K [22], where the sign of magnetoelastic coupling changes. Disappearance of residual FM phase (for heating from below $T_{C}$ ) or FM nuclei (for thermal cycling within the temperature range of AFM phase existence) at Villari point was suggested as feasible mechanism of abrupt vanishing of thermal hysteresis. Dislocations, possessing intense strain fields, were suggested as defect species, stabilizing FM phase [21]. In this work we use internal friction (IF) technique to further study thermal hysteresis in polycrystalline Dy.

\section{EXPERIMENTAL TECHNIQUE}

Samples with dimensions of $1.0 \times 1.3 \times 12.2 \mathrm{~mm}^{3}$ were spark cut from a sheet of polycrystalline dysprosium of 99.9 wt.\% purity, supplied by Sigma Aldrich. Data will be shown for as received (rolled) state of material. The results for annealed samples and comparison with the as-received state are given in [6].

The piezoelectric ultrasonic composite oscillator operating at a frequency around $90 \mathrm{kHz}$ was used to measure the IF, $\delta$, and Young's modulus (YM), $E$, between 80 and $300 \mathrm{~K}$. A detailed description of the method and the experimental setup can be found elsewhere [23]. Oscillatory strain amplitude was stabilized in all experiments at $10^{-5}$. Measurements were done in zero external magnetic field and under axial magnetic field $H=9 \mathrm{kA} / \mathrm{m}$. Measurements of the IF and YM temperature spectra were performed under three different protocols: 1) continuous cooling/heating, when the IF spectra are registered between $290 \mathrm{~K}$ and $95 \mathrm{~K}$ (in the AFM phase) or $290 \mathrm{~K}$ and $80 \mathrm{~K}$ (including the range of AFM-FM transition) under constant cooling and heating rate. Heating/ cooling rates of $2,0.75$, and $0.25 \mathrm{~K} / \mathrm{min}$ were used; 2) discontinuous mode, with interruptions at sev-

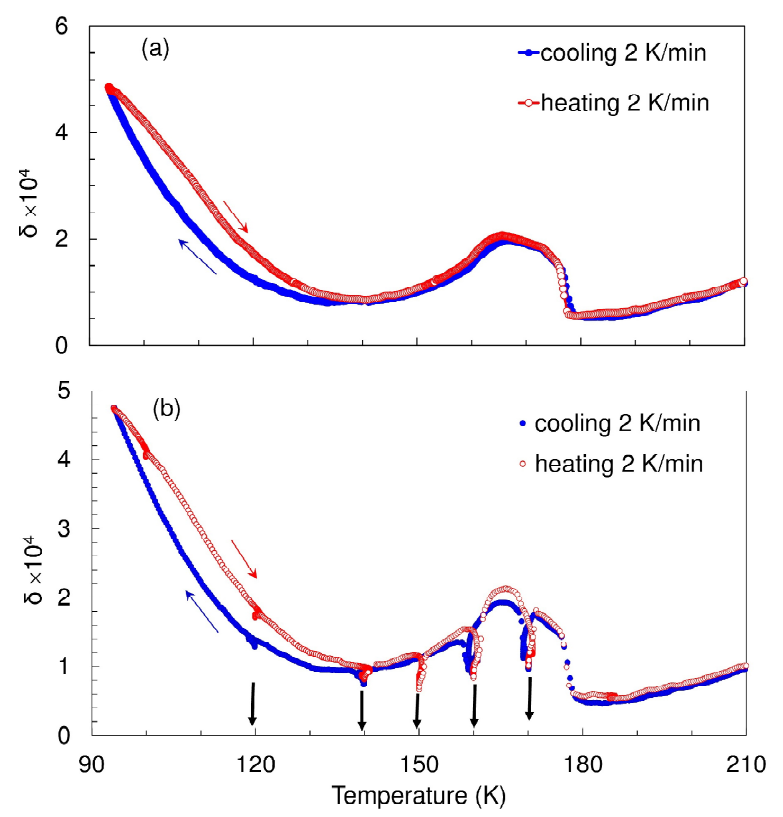

Fig. 1. Internal friction, $\delta$, on cooling to $95 \mathrm{~K}$ and subsequent heating at $2 \mathrm{~K} / \mathrm{min}$ : (a) continuous scan; (b) measurements with interruptions on cooling and heating at $100,120,140,150,160,170$, and $185 \mathrm{~K}$ (temperatures are marked by vertical arrows) for 40 min of isothermal dwelling. Oscillatory strain amplitude $\varepsilon_{0}=10^{-5}$.

eral selected temperatures on heating/cooling for 40 min of isothermal dwelling; 3) continuous mode under magnetic field $H=9 \mathrm{kA} / \mathrm{m}$ during thermal cycling in the AFM phase.

\section{RESULTS AND DISCUSSION}

Fig. 1 shows IF $(\delta)$ versus temperature on cooling to 95K and subsequent heating. During cooling, the IF remains rather low in the paramagnetic phase and decreases gradually down to rather low logarithmic decrement values ca. $5 \cdot 10^{-5}$ close to $T_{N}$, Fig. 1a. At $T_{\mathrm{N}}$ the IF increases sharply by a factor of four and continues to increase on further cooling until a maximum is reached at a temperature of Villari point $T_{\mathrm{V}}=166 \mathrm{~K}$. An abrupt increase of the IF during AFM ordering, instead of an IF peak [24,25], is a superposition of a critical fluctuations peak, somewhat suppressed in the as received samples [6] due to high density of defects (dislocations) and rather high IF in the AFM state. The latter is associated with AFM DWs [24]: the frequency used $\sim 90 \mathrm{kHz}$ is too low to detect resonant/relaxation phenomena of individual spins and corresponds to a maximum sensitivity to such cooperative effect as motion of domain walls in FM materials [26]. Fig. 1b shows the IF temperature spectra with interruptions of cool- 


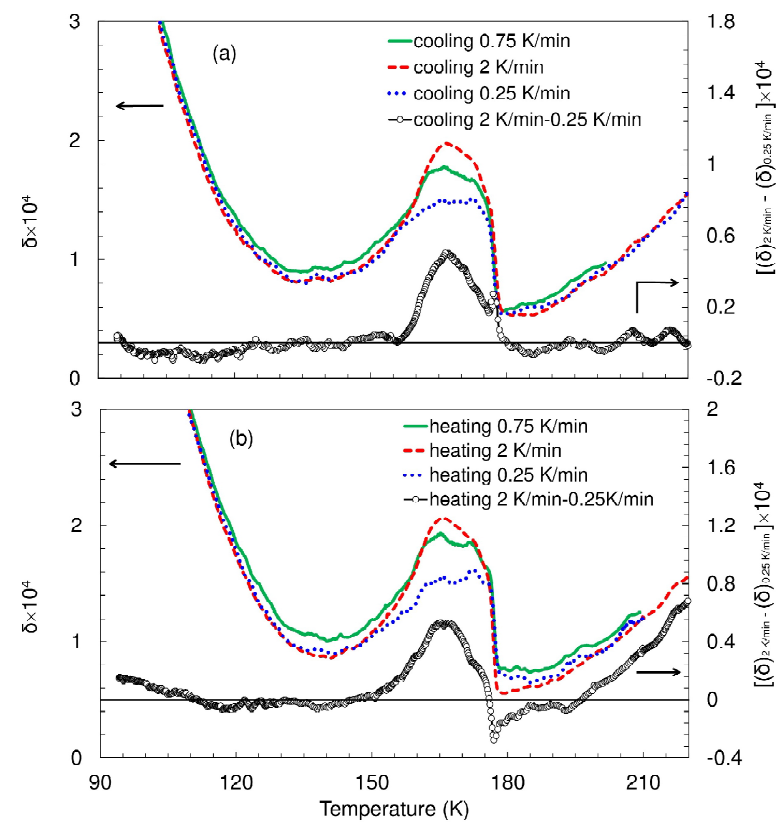

Fig. 2. Internal friction, $\delta$, in thermocycles $250 \mathrm{~K}-$ $95 \mathrm{~K}-250 \mathrm{~K}$ performed under cooling-heating rates of $2 \mathrm{~K} / \mathrm{min}, 0.75 \mathrm{~K} / \mathrm{min}$, and $0.25 \mathrm{~K} / \mathrm{min}$ : (a) cooling (b) heating; difference between spectra at $2 \mathrm{~K} / \mathrm{min}$ and $0.25 \mathrm{~K} / \mathrm{min}$ is shown as well. Oscillatory strain amplitude $\varepsilon_{0}=10^{-5}$.

ing/heating and isothermal dwellings at several temperatures. Isothermal dwellings above the Néel temperature (185K) and at 100 and $120 \mathrm{~K}$ do not show any time dependence of the IF. According to Fig. $1 \mathrm{~b}$, the high IF value below $T_{\mathrm{N}}$ is formed by a nonisothermal IF, which drops nearly 3 times after isothermal dwelling for $40 \mathrm{~min}$ in the range between 160 and $170 \mathrm{~K}$. The broad IF maximum at the temperature of Villari point disappears after isothermal dwelling. Following [6], the non-isothermal IF consists of $\dot{T}$-dot and time-dependent terms, corresponding respectively to the "fast" and "slow", glasslike dynamics of DWs. The "fast" IF term has been attributed to an ultrasonic transitory damping term, which scales with $\dot{T}$-dot and is related to translatory motion of DWs either under the thermal stresses or as a consequence of the variation of the pitch of the AFM spiral with temperature [6].

To confirm the existence of $\dot{T}$-dot dependent transitory IF component, IF temperature spectra in the present work were registered under different cooling/heating rates. Fig. 2 shows such spectra at 2 , 0.75 , and $0.25 \mathrm{~K} / \mathrm{min}$ and the difference between the spectra at $2 \mathrm{~K} / \mathrm{min}$ and $0.25 \mathrm{~K} / \mathrm{min}$. In accordance with our previous conclusion [6], the maximum of the $T$-dependent IF transitory term is located in the Villari point $T_{V}=166 \mathrm{~K}$. Fig. 2 also indicates the
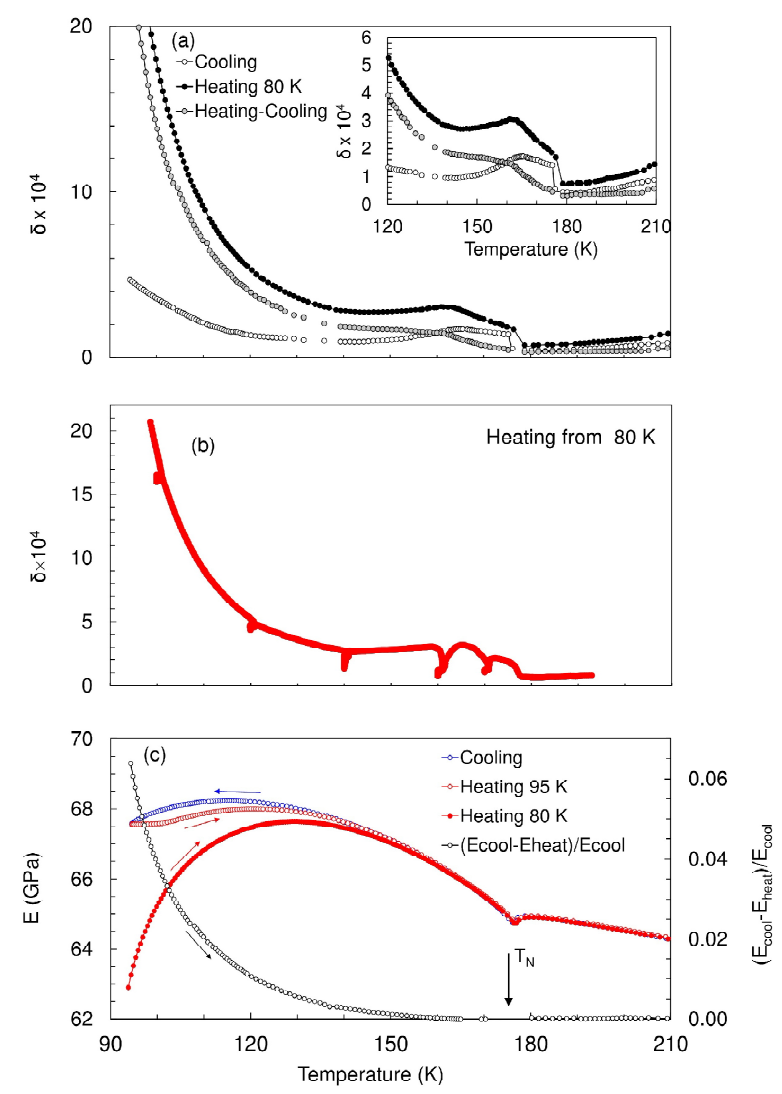

Fig. 3. Temperature spectra of the internal friction, $\delta,(\mathrm{a}, \mathrm{b})$ and Young's modulus, $E,(\mathrm{c})$ in a continuous $(\mathrm{a}, \mathrm{c})$ and interrupted for $40 \mathrm{~min}$ of isothermal dwelling (b) temperature cycle $210-80-210 \mathrm{~K}$. The differences between the values of internal friction (a) and Young's modulus (c) on heating and cooling are shown. Panel (c) shows also the Young's modulus in a temperature cycle 210-95-210K. Oscillatory strain amplitude $\varepsilon_{0}=10^{-5}$.

range between $150 \mathrm{~K}$ and $T_{\mathrm{N}}$, wherein the "fast" IF component persists.

The IF diminishes below $166 \mathrm{~K}$ and raises again starting from approximately $140 \mathrm{~K}$; the IF does not show any time dependence below this temperature, Fig. 1b. Figs. $1 \mathrm{a}$ and $1 \mathrm{~b}$ indicate that thermal hysteresis exists not only during heating the sample from the FM phase [16-20], but even during thermal cycling within the AFM phase. It is worth mentioning that thermal hysteresis for thermal cycles within AFM phase shows an upper limit around 140K, Fig. 1 , the temperature where a notable rise of the IF is detected on cooling. In that case, the temperature of $140 \mathrm{~K}$ serves as a characteristic point. Fig. 3 shows IF and $Y M(E)$ temperature spectra for a thermocycle with the lower temperature of $80 \mathrm{~K}$, i.e. in the FM state. A comparison of thermal cycles in Figs. 1 and 3 reveals the following details: 


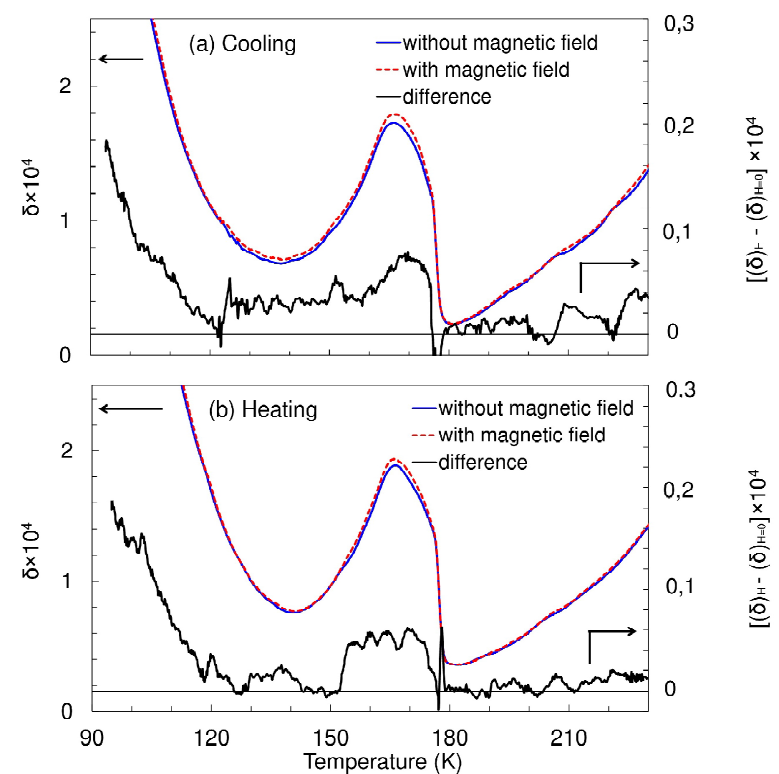

Fig. 4. Temperature spectra of internal friction, $\delta$, in thermocycles $250 \mathrm{~K}-95 \mathrm{~K}-250 \mathrm{~K}$ without magnetic field, $H=0$, and under magnetic field $H=9 \mathrm{kA} / \mathrm{m}$ : (a) cooling, (b) heating. The difference between the spectra with $H=9 \mathrm{kA} / \mathrm{m}$ and $H=0$ is also shown. Oscillatory strain amplitude $\varepsilon_{0}=10^{-5}$.

- The IF hysteresis is much more prominent in the temperature cycle to the FM phase. The difference between IF spectra on cooling and heating can be detected up to the Néel temperature, and shows three distinct stages, Fig. 3a: i) fast decrease upon heating to ca. $140 \mathrm{~K}$; ii) leveling off between 140 and 166K; iii) an abrupt decline above 166K;

- The hysteresis in elastic properties (Young's modulus), Fig. 3c, also is much more intense after cooling the sample to the FM phase. However, in contrast with the IF, the difference between YM values in cooling and heating scans does not show characteristic temperatures and declines monotonously down to the Villari point at $166 \mathrm{~K}$;

- Fig. 3b shows that the non-isothermal IF component is predominant during heating from $80 \mathrm{~K}$ for temperatures above approx. 140K. This is the component responsible for a plateau of the difference between heating and cooling scans between 140 and 166K, Fig. 3a.

The properties of thermal IF and YM hysteresis are consistent with the interpretation, suggested in [21] based on studies of reversible inverse magnetostriction and magnetic susceptibility and on discovery of the ultrasonic transient damping in polycrystalline Dy [6]. More specifically, thermal hysteresis upon heating from the FM phase of the reversible inverse magnetostriction and magnetic susceptibility vanished at the temperature of Villary point and was attributed to the existence of residual FM phase, stabilized by lattice defects up to the Villari point in which the sign of magnetoelastic coupling changes the sign. Data in Figs. 3a-3c confirm the crucial role of the Villari point in the IF and YM hysteresis. However, contrary to the reversible magnetostriction and susceptibility thermal hysteresis, the IF hysteresis does not disappear at the Villari point. This difference is natural, taking into account that the IF at frequencies around $10^{5} \mathrm{~Hz}$ includes a strong contribution from the dynamics of DWs [6]. Fig. 3b indicates that the IF hysteresis that persists above the Villari point is formed by non-isothermal IF component, attributed to translatory motion of DWs [6].

A new observation is the existence of IF and YM hysteresis, although only minor compared with the hysteresis observed on heating from the FM phase, even for thermal cycles restricted to the AFM phase. A feasible interpretation of this hysteresis can be associated with nucleation of FM phase, below approximately $140 \mathrm{~K}$. This hypothesis implies the existence of minor amount of FM phase starting from $140 \mathrm{~K}$ and an increase of this amount at lower temperatures. Hence, typical features of magnetomechanical damping should emerge over this temperature range, like macroeddy current damping, promoted by applied field. The present high-resolution acoustic technique allows us to detect these expected minor IF variations under applied field. Fig. 4 shows a comparison of temperature spectra of the IF registered without magnetic field and under field of $9 \mathrm{kA} / \mathrm{m}$. As expected, the applied field promotes damping (albeit rather weakly due to presumably very low amount of FM nuclei) starting from ca. $130-140 \mathrm{~K}$ and the effect of magnetic field intensifies rapidly below this temperature. Since the frequency of experiments used is perfectly suited to detect macroeddy current damping [27], the observed enhancement of the IF by applied field can be attributed to the emergence of the net magnetization of the sample due to nuclei of the FM phase. Surprisingly, applied magnetic field affects the IF spectra in the vicinity of the Villari point, as shown in Fig. 4. The origin of this effect requires further research.

\section{CONCLUSIONS}

- Thermal hysteresis is detected in polycrystalline Dy even during cycling within antiferromagnetic phase and is attributed to the formation and disap- 
pearance of nuclei of ferromagnetic phase below approximately $140 \mathrm{~K}$.

- Data on internal friction and Young's modulus confirm the hypothesis that the origin of thermal hysteresis in Dy (and other rare earth elements and alloys) is residual ferromagnetic phase, stabilized by lattice defects.

- Villari point in polycrystalline Dy is a characteristic temperature, defining the behaviour of thermal hysteresis of internal friction and Young's modulus.

\section{ACKNOWLEDGEMENTS}

The work was supported by the Ministry of Education and Science of the Russian Federation, Goszadanie No 3.1421.2017/4.6

\section{REFERENCES}

[1] T. Jungwirth, J. Sinova, A. Manchon, X. Marti, J. Wunderlich and C. Felser // Nature Phys. 14 (2018) 200.

[2] R. Duine // Nature Mater. 10 (2011) 344.

[3] B. G. Park, J. Wunderlich, X. Martí, V. Holý, Y. Kurosaki, M. Yamada, H. Yamamoto, A. Nishide, J. Hayakawa, H. Takahashi, A. B. Shick and T. Jungwirth // Nature Mater. 10 (2011) 347.

[4] P. Wadley, B. Howells, J. Zelezny, C. Andrews, V. Hills, R. P. Campion, V. Novak, F. Freimuth, Y. Mokrousov, A. W. Rushforth, K. W. Edmonds, B. L. Gallagher and T. Jungwirth // Science 351 (2016) 587.

[5] O. Gomonay, T. Jungwirth and J. Sinova // Phys. Rev. Lett. 117 (2016) 017202.

[6] S. Kustov, lu. Liubimova, M. Corró, J. TorrensSerra, X. Wang, C. R. S. Haines and E. K. H. Salje, Temperature Chaos, Memory Effect, and Domain Fluctuations in the Spiral Antiferromagnet $D y$, unpublished.

[7] S. W. Chen, H. Guo, K. A. Seu, K. Dumesnil, S. Roy and S. K. Sinha // Phys. Rev. Lett. 110 (2013) 217201.

[8] J. F. Elliott, S. Legvold and F. H. Spedding // Phys. Rev. 94 (1954) 1143.

[9] K. P. Belov, R. Z. Levitin, S. A. Nikitin and A. V. Ped'ko // Soviet Phys. JETP 13 (1961) 1096.
[10] E. B. Amitin, V. G. Bessergenev and Y. A. Kovalevskaya // Soviet Phys. JETP 57 (1983) 117.

[11] C. Isci and S. B. Palmer // J. Phys. F: Met. Phys. 8 (1978) 247.

[12] A. V. Andrianov, Y. P. Gaidukov, A. N. Vasil'ev and E. Fawcett // J. Magn. Magn. Mater. 97 (1991) 246.

[13] A. S. Chernyshov, A. O. Tsokol, A. M. Tishin, K. A. Gschneidner and V. K. Pecharsky // Phys. Rev. B71 (2005) 184410.

[14] M. T. Alkhafaji and N. Ali // J. Alloys Compd. 250 (1997) 659.

[15] M. K. Wilkinson, W. C. Koehler, E. O. Wollan and J. W. Cable // J. Appl. Phys. 32 (1961) S48.

[16] S. Kobayashi // Phys. Rev. Lett. 106 (2011) 057207.

[17] A. Del Moral and E. W. Lee // J. Phys. F: Met. Phys. 4 (1974) 280.

[18] T. J. Mckenna, S. J. Campbell, D. H. Chaplin and G. V. H. Wilson // Phys. status solidi (a) 75 (1983) 421.

[19] M. Rosen // Phys. Rev. 174 (1968) 504.

[20] F. J. Darnell // Phys. Rev. 130 (1963) 1825.

[21] I. Liubimova, M. Corró Moyà, J. Torrens-Serra, V. Recarte, J. Pérez-Landazábal and S. Kustov // Metals 7 (2017) 215.

[22] M. L. Corró, A. El Hichou, E. Cesari and S. Kustov // J. Phys. D: Appl. Phys. 49 (2016) 015001.

[23] G. Gremaud, S. Kustov and O. Bremnes, In: Mechanical Spectroscopy Q-1 2001 with Applications to Materials Science, ed. by R. Schaller, G. Fantozzi and G. Gremaud (Trans. Tech. Publications Ltd, 2001), p. 652.

[24] B. Lüthi, In: Physical Acoustics in the Solid State, ed. by M. Cardona (Springer-Verlag Berlin Heidelberg, 2007), p. 93.

[25] R. J. Pollina and B. Lüthi // Phys. Rev. 177 (1969) 841.

[26] W. P. Mason // Phys. Rev. 83 (1951) 683.

[27] J. Torrens-Serra, F. Solivelles, M. L. Corró, M. Stoica and S. Kustov // J. Phys. D: Appl. Phys. 49 (2016) 505003. 